\title{
Special Issue on Emergent Effects in Stochastic Neural Networks with Application to Learning and Information Processing
}

Containing a selection of extended papers from the 15th Granada Seminar, Spain, September 2019

Joaquín J. Torres
, Miguel A. Muñoz
Mejías $^{\mathrm{e}}$

${ }^{a}$ Departamento de Electromagnetismo y Física de la Materia and Instituto Carlos I de

Física Teórica y Computacional, Universidad de Granada, 18071 Granada, Spain

${ }^{b}$ Computational Neuroimaging Group, Biocruces-Bizkaia Health Research Institute. Barakaldo, Spain

${ }^{c}$ Ikerbasque - the Basque Foundation for Science, Bilbao, Spain

${ }^{d}$ Cell Biology and Histology Department, University of the Basque Country (UPV/EHU). Leioa, Spain

${ }^{e}$ Swammerdam Institute for Life Sciences, University of Amsterdam, 1090GE

Amsterdam, Netherlands

This special issue includes a series of 12 articles, which represent a selection of extended contributions presented at the 15th Granada Seminar on Computational and Statistical Physics held in Granada from September 17 to 20, 2019, and organized by Institute Carlos I for Theoretical and Computational Physics at the University of Granada.

The brain is a paradigmatic example of a highly complex system, in which cognitive functions are the result of emergent phenomena derived from collective effects of a large number of microscopic elemental components, such as neurons, synapses and glial cells, which in turn interact with multiple elements at higher spatial scales, thus forming microcircuits or anatomical structures with a well-characteristic cellular, functional and organizational differentiation.

It is precisely for this reason that the tools and ideas of from statistical mechanics and the modern field of complex-networks provide rigorous and adequate frameworks to shed light on the collective characteristics of brain 
networks, thus opening a window of opportunity to investigate the theory of concomitant cognitive functions. Theoretical advances in this regard are now crucially complemented by the astonishing availability of both structural and functional data, on a wide range of spatial and temporal scales, as provided by recent developments in neuroimaging and neurophysiology.

Despite the large number of scientific publications on the structure and dynamics of brain networks and, in particular, on the interaction of them with learning and information processing, still today there are unsolved challenges, including aspects related to the acquisition and consolidation of memory, the emergence of high-level cognition, the plasticity and reconfiguration of these networks to compensate for cerebral damage, to name just a few. The use of computers has proven to be an extremely powerful tool for modeling neural activity, synaptic transmission, as well as designing biologically inspired circuits.

Therefore, we are currently in a position to achieve a much deeper understanding of how the brain works and how the large repertoire of high-level functionalities emerge. This combination of emerging neural properties and complex brain networks, understood from a computational point of view, and with applications to artificial intelligence and computer science, was the focus of the latest 15th edition of the Granada Seminar on Computational and Statistical Physics. This conference constituted a meeting point where the latest advances in neuroscience, computational modeling and research in neural networks were presented in a highly interdisciplinary and stimulating environment. This special issue gathers together various high-quality original contributions presented at this seminar, covering the areas of research in experimental neuroscience, computational neuroscience, artificial intelligence and brain networks. In particular, the list of articles includes the following contributions:

In the first work, Zambrano et al. extend the most common algorithms and computational frameworks for artificial neural networks to a more biological and realistic scenario. In particular, the authors present a biologically plausible continuous-time reinforcement learning (RL) framework appropriate for continuous temporal representations. The authors apply this framework to the modeling of working memory tasks with the addition of an action-selection system inspired by the functioning of the basal ganglia, a novel exploratory mechanism, and an accessory network to model feedback with transmission delays. This novel RL algorithm enables neural networks to accumulate evidence efficiently over time, leading to levels of performance 
and reaction times similar to those obtained in experiments in behavioral neuroscience.

Detecting specific signatures of neural activity, such as spike correlations, is an especially important task that can be difficult in stochastic neural networks. The problem is often computationally intensive and may require carefully assembled strategies to analyze the parallel activity of multiple interconnected neurons. In this issue, Budzinski et al. present a novel approach to detect burst synchrony in a small-world network of neurons modeled as Rulkov iterated maps. The approach is based on a symbolic method of time series analysis, the ordinal analysis, which is suitable for detecting non-linear temporal correlations. Budzinski et al. show that the ordinal analysis uncovers different dynamic regimes and fine details of the bursting activity of cells, which are not captured by other methods. The impact of network properties, such as coupling strength and topology, is also evaluated. Overall, the method can be applied to raw data and therefore offers an alternative and less computationally intensive approach to detect synchronous dynamics in neural networks.

One of the most relevant characteristics of the stochastic activity of neural networks, both in experiments and in computational models, is the existence of long temporal correlations which often reflect scale-free avalanches of neural activity. Alterations of such correlations might correspond to pathological states of brain activity. The characterization of the temporal correlations is therefore vital to verify the hypothesis of the brain operating close to a critical point. In this issue, Lombardi et al. present a study of temporal correlations in the avalanche sequences of broadband activity of human EEG and MEG recordings during resting state. The authors report the existence of long-range power-law correlations, with power-law exponents which are consistent across EEG and MEG data and robust to different scaling parameters and temporal binning. This study constitutes a preliminary step to develop approaches for inferring long-range correlations in extended neural systems, and to identify pathological behaviors in the complex interplay of cortical rhythms.

It is widely accepted that elements of neural systems, such as neurons and synapses, can have their dynamics affected by different sources of noise. Likewise, it is also known that there are intrinsic mechanisms that take advantage of this stochasticity to determine neuronal function. An example is the stochastic resonance (SR) phenomenon, where adding a certain level of non-zero noise to a non-linear system reduces information loss. In this issue, 
S. Ikemoto investigates neural networks consisting of thresholding functions that exploit SR at run time and during training, with the goal of smooth mapping and back propagation. Such a neural network can properly operate only when noise is present. In particular, the author focuses his study on a neural network for which only a sub-network is activated selectively by adding noise locally on that sub-network, introducing a new activation function. The result is that this noise-activated sub-network becomes a functionalized neural network capable to train and map inputs to outputs. Interpolations are further investigated by imposing varying noise intensity on various regions of the network after subnetworks are trained separately.

An interesting aspect about nonlinear dynamics with application in neuroscience, robotics and artificial intelligence refers to the study of the so-called Central Pattern Generators (CPG). These are neural circuits that generate robust sequences of coordinated neural activity to control motor rhythms. In this issue, Garrido-Peña et al. study the characteristics of the dynamical invariants in the relationships between specific intervals that construct the CPG sequence and the instantaneous period of the rhythm in the pyloric CPG of crustacean. More precisely, they analyze different aspects concerning the variability of the intervals comprising the rhythm in a model of a mollusk feeding CPG under different neuron stimulation. The authors report dynamical invariants found only for specific time intervals building the sequence of the feeding $\mathrm{CPG}$, and conclude that such dynamical invariants can be a universal feature of any sequence generating circuit.

As previously stated, the dynamical control of different rhythms on CPGs has also important application in robotics. Recently the use of hierarchical networks in motor pattern generation in hexapod robots and in insect movement has provided ways for their understanding. In this issue, Barrio et al. analyzed in deep the dynamics of a six coupled neurons CPG of insect movement that produces the global leg coordination pattern. The authors provide a detailed study without further simplifications of the possible gaits patterns generated by the CPG using numerical techniques recently developed for the study of small networks. They found that most of the observed patterns (symmetric and non-symmetric) follow routes which lead to a stable tripod gait. Since the model includes many parameters, an algorithm to locate the limit of the tripod gait in the parameter space is also introduced, which shows that there is a large three parametric region where the tripod pattern is ubiquitous and highly dominant in rapidly moving insect regimes.

Other important aspect in neuroscience concerns with the study of syn- 
chronization phenomena and their relation, e.g., with some brain functions and pathological conditions. In this issue, Ódor et al. studied the prototypical model for phase synchronization, the Kuramoto model with Gaussian noise, running on empirically obtained high-resolution human structural networks (the KKI-18 large human connectome graph). The authors reveal that the distributions of de-synchronization times exhibit power-law tails, characterized exponents compatible with those measured in vivo, and analyze a number of other features by comparing the results with those emerging on other network topologies, thus putting the emphasis on how the structure influences the emerging function.

Reservoir Computing is a fascinating approach in machine learning that combines high computational capabilities with an easy offline learning algorithm. As shown in the contribution by Morales et al. in this issue, the performance of traditional reservoir approaches - such as "Echo state networks" (ESN) - can be significantly enhanced by introducing biologically-inspired plasticity rules. More specifically, when assessing nonlinear time-series prediction tasks, the authors concluded that this improvement can be associated with a reduction on pairwise correlations among units, with optimal performance achieved if a combination of diverse forms of plasticity (synaptic and non-synaptic rules) is implemented. Their results also suggest that optimal reservoirs seem to emerge at the edge of instability, thus creating a new scenario to test and prove the inspiring hypothesis that biological computing systems could operate at the "edge of chaos".

Also in this issue, Apicella et al. extended the study of directed linear chains of neurons (where one neuron only connects to the previous one and the next in the chain) by adding heterogeneity in the connections and introducing long-range connectivity. The authors present simulations and non-linear stability analyses to characterise the amplification properties of a signal input introduced into the system, and provide stability phase diagrams together with the report of detailed emergent and collective synchronization patterns. In their article the authors also determine the relationship between the non-local coupling strength distribution and the frequency of the network oscillations and illustrate how the presence of long-range interactions introduces novel and rich dynamical behavior into the system, such as synchronous oscillations and phase coherence among all the interacting units.

The complex interplay between spontaneous spiking activity and shortterm synaptic plasticity in neural networks can lead to intriguing phenomena with strong computational implications. In this issue, Zendrikov and 
Paraskevov reported the emergence of spontaneous repetitive synchronization in the population spikes as a consequence of such interplay where synaptic plasticity nonlinearly is modulating the interaction between neurons, which induces different emerging phenomena depending on network topology. For large-scale two-dimensional networks with small-world network connectomes, periodic population spikes in the form of circular traveling waves diverging from seemingly non-stationary nucleation sites can emerge. Moreover, the mechanism behind this phenomenon is different form stochastic resonance and could be related with transient chimera patterns.

Functional (correlation) networks provide us with a description of activity patterns in the brain. The hierarchical and modular structure of such networks has been associated with optimal balance between segregated tasks and integrated, whole-brain, function. On the other hand, it is well-known that the underlying structural networks of neural connectivity are organized in a hierarchical and modular way and thus, one can wonder which types of dynamical regimes can generate hierarchical-modular functional networks when operating on such structural networks. In the present issue, Safari et al. show - by profusely exploiting tools of spectral graph theory - that these features can emerge in the neighbourhood of critical points for the dynamical processes regulating neural activity. In particular, the authors study the persistence and collapse of hierarchical modular functional networks and reveal that they emerge in the quasi-critical regime (associated with optimal processing capabilities and normal brain function), while they break down in other dynamical regimes (often associated with pathological conditions). These results offer important clues for the study of optimal neurocomputing architectures and processes.

Finally, Fernandez-Iriondo et al. present in this issue a study concerning the relationships between structural brain connectivity (SC), which is considered static and does not change for at least several months of our life, and dynamic functional connectivity (DFC), which changes very quickly, even faster than one second. The authors introduce two metrics for network comparison: the similarity between SC and DFC, and the intrinsic time-variability of DFC. Overall, the authors found that the cerebellum, as compared to the cerebral cortex, is a key structure in the relation of $\mathrm{SC}$ and DFC. These results expand current knowledge on cerebellar networks, which are extremely rich and complex, and participate in multiple cognitive tasks such as movement control and coordination, executive function and emotional regulation. 
The Guest editors would like to thank the Editors-in-Chief of Neurocomputing for giving us the opportunity to edit this issue, as well as to the Neurocomputing editorial assistant. We wish to thanks also the reviewers who have assessed the articles in this issue. We hope that Neurocomputing readers find this issue useful.

The Guest Editors

\section{Acknowledgments}

JJT and MAM acknowledge financial support from the Spanish Ministry of Science and Technology, and the Agencia Española de Investigación (AEI) under grant FIS2017-84256-P (FEDER funds) and from the Consejería de Conocimiento, Investigación y Universidad, Junta de Andalucía and European Regional Development Fund (ERDF), with reference SOMM17/6105/UGR. JMC acknowledges financial support from spanish Ministerio Economia, Industria y Competitividad and FEDER (grant DPI2016-79874-R) and from the Department of Economical Development and Infrastructure of the Basque Country (Elkartek Program, KK-2018/00032 and KK-2018/00090). JFM acknowledges financial support from the European Union's Horizon 2020 Framework Program for Research and Innovation under the Specific Grant Agreement No. 945539 (Human Brain Project SGA3). 